\title{
Tratamiento de la Maloclusión Clase III en el paciente pediátrico. Reporte de caso
}

Pabón- $A M^{1}$

Aristizábal-LM ${ }^{1}$

Hernández-J $A^{2}$

\section{Resumen}

El tratamiento temprano de las alteraciones oclusales y esqueléticas devuelve la armonía y funcionalidad al complejo craneofacial, simplifica los procedimientos correctivos, reduce las necesidades de exodoncias de dientes permanentes y trae beneficios psicológicos a los pacientes, así mismo provee las condiciones necesarias para que cada paciente exprese su potencial de crecimiento.

Es importante el tratamiento de estas maloclusiones tan pronto se identifican, puesto que ayudaran a un mejor manejo. La máscara facial de protracción es una excelente opción para el tratamiento de la maloclusión clase III especialmente si se combina con expansión rápida del maxilar y se continua con AOF.

En este artículo presentamos la terapia realizada a un paciente de 7 años con maloclusión clase III esquelética y la evolución del caso durante seis años.

Palabras clave: enfermedades maxilomandibulares, niños, maloclusión.

Relato de caso

\section{Tratamento da má oclusão de Classe III no paciente pediátrico. Relato de caso}

\section{Resumo}

O tratamento precoce das alterações oclusais e esquéleticas devolve a harmonia e a funcionalidade ao complexo craniofacial, simplifica os procedimentos corretivos, reduz a necessidade de exodontias de dentes permanentes e traz benefícios psicológicos ao paciente. Além disso, o tratamento mantém as condições necessárias para que o paciente expresse todo o seu potencial de crescimento.

A máscara facial de protração é uma excelente escolha para o tratamento de classe III, especialmente se associada à disjunção palatal rápida seguida de tratamento funcional.

Nesse artigo, apresentamos o tratamento realizado em um paciente de 7 anos com maloclusão 
classe III esquelética e a evolução do caso durante 6 anos.
Palavras-chave: Doenças Maxilomandibulares, maloclusão classe III, máscara facial de protração.

\section{Case report}

\section{Treatment of Class III Malocclusion in pediatric patient. A case report}

\begin{abstract}
Early treatment of skeletal and occlusal disorders improve the harmony and function of the craniofacial complex. Corrective procedures, reduces the need for extractions of permanent teeth, and gives psychological benefits to the patients. In addition, it provides the patient's condition to express his potential growth. The face protraction mask is an excellent choice for the treatment of class III when combined with rapid maxillary expansion and continues functional oral appliance treatment.
\end{abstract}

In this article we present the therapy to a patient of 7 years old with class III malocclusion and the evolution of the case for six years.

Keys words: Jaw diseases, class III malocclusion, face protraction mask.

\section{Introducción}

Unos de los papeles fundamentales de la odontología pediátrica es guiar la erupción y el desarrollo de la dentición primaria, mixta y permanente. El principal objetivo es conservar una dentición que esté libre de caries, con tejidos periodontales sanos y funcionando adecuadamente con una oclusión armónica, equilibrada y estética.

Cuando utilizamos el término de mordida cruzada anterior nos referimos a una anomalía en el plano antero- posterior donde los dientes inferiores están delante de los dientes superiores y puede tener un componente dentoalveolar, funcional o esquelético.

La mordida cruzada anterior dentoalveolar se puede dar a causa de patrones de erupción lingual y/o erupción retardada de incisivos superiores, inclinación labial de incisivos inferiores, presencia de dientes supernumerarios y longitud inadecuada del arco dental entre otras.

Cuándo la mordida cruzada anterior tiene un componente funcional que posiciona la mandíbula en cierre en una posición anterior respecto al maxilar superior se denomina seudoclase III, este movimiento es por lo general el resultado de contactos dentales prematuros que provocan el desplazamiento.

La mordida cruzada anterior puede estar presente en pacientes con maloclusión clase III con componente esquelético asociada con discrepancias en el tamaño y / o posición de los maxilares.

Las maloclusiones clase III son alteraciones que pueden ser causadas por una retrusión maxilar o un prognatismo mandibular o una combinación de las dos ${ }^{1}$. Tsai en un estudio reciente examino 32 niños coreanos con mordida cruzada anterior en la dentición temporal y los comparó cefalométricamente con 32 niños control, los niños con mordida cruzada anterior presentaron una base craneal anterior pequeña, retrusión maxi- 
lar con un desarrollo normal de la mandíbula, desarmonía anteroposterior maxilo-mandibular y posición labial de los incisivos ${ }^{2}$. Cuando los pacientes con maloclusión clase III se examinan cefalométricamente, pueden existir numerosas combinaciones de la posición esqueletal y/o dental. A pesar que la retrusión maxilar es el hallazgo más frecuente, la etiología estructural real puede ser difícil de identificar a una edad temprana, como lo confirma A Yagci y col. quien dice que en la mayoría de los casos de maloclusión clase III está caracterizada por deficiencia del maxilar en un $60 \%{ }^{1}$.

Según Yang el 40\% al 50\% de los pacientes ortodoncicos en Corea tiene tratamiento de maloclusión clase III. Kitai y col. reportaron que alrededor del 5 al 20\% de la población japonesa presentan maloclusión clase III, Y Jonson y col. establecieron que el 23\% de los niños chinos tiene maloclusiones clase III ${ }^{3}$. En general se ha establecido que esta alteración oclusal es bastante común en pacientes asiáticos ${ }^{4,5}$. En americanos europeos es de $0,8 \%$ y en afroamericanos esta entre el 0,6 y el 1,2\%. En cuanto a los componentes esqueléticos de una clase III, se ha reportado que la retrusión esquelética maxilar ocurre más en caras de asiáticos, mientras que el prognatismo mandibular se observó más en individuos de ancestros europeos americanos ${ }^{4}$. En Colombia en un estudio realizado en el año 2001 por Thilander y col. se encontró una prevalencia de mordidas cruzadas anteriores del 5,8\% en una población de 4724 niños entre los 5 y 17 años de edad, y de clases III en la misma población del $3,7 \%$. Las diferencias se atribuyen a la existencia de mordidas cruzadas de tipo funcional en un $2,1 \%$ por actividad muscular ${ }^{5}$.

En el tratamiento temprano de la maloclusión clase III se pretende crear un ambiente en donde se puede dar un desarrollo dentofacial fa- vorable para prevenir cambios progresivos en tejidos duros y blandos, mejorar las diferencias esqueletales y brindar condiciones optimas para el futuro crecimiento craneofacial. Este manejo temprano beneficia la estética y el perfil facial, previene la recesión periodontal y el desgaste dental, brinda una mejoría funcional de la ATM, reduce los efectos psicosociales negativos en los niños y disminuye las necesidades de la cirugía ortognática simplificando las fases terapéuticas posteriores $^{6,7}$.

Las opciones de tratamiento varían dependiendo del diagnóstico y de factores como la edad y el estadío de maduración ósea en la que se encuentre el paciente. Es reconocido que las estructuras óseas y neuromusculares responden mas rápida y eficientemente a la terapia cuanto más joven sea el organismo ${ }^{8}$.

Una variedad de métodos de tratamiento ha demostrado ser efectiva con cambios estadísticamente significativos en el complejo craneofacial en pacientes con mordidas cruzadas anteriores y maloclusiones clase III $^{9}$. Entre las alternativas de tratamiento esta el uso de agregados de resina o pistas directas en dientes primarios; planos inclinados, aparatología ortopédica funcional, ortopedia extraoral, ortodoncia correctiva y la cirugía ortognática; el uso de cada alternativa terapéutica dependerá de la edad cronológica, dental y ósea del paciente así como de la preferencia del profesional tratante entre otros factores. En este artículo el tratamiento de elección fue la máscara facial de protracción debido a las características dentoesqueletales del paciente del cual se reporta el caso.

\section{La Máscara Facial de Protracción}

Es una de las mejores opciones para el tratamiento temprano de una maloclusión clase III en de- 
sarrollo. Fue desarrolla por Delaire (1971, 1976) y modificada por Petit (1982) cambiando la forma del marco de alambre que une las superficies de anclaje, creando dinamismo, aumentando la magnitud de la fuerza generada por el aparato, reduciendo así el tiempo de tratamiento global; luego en 1987, Mc Namara introdujo el uso de un aparato de expansión adherida con cobertura oclusal de acrílico (férula adherida) para la protracción maxilar ${ }^{10}$. La estructura craneofacial en los niños es muy maleable y con el uso de esta máscara se pueden producir cambios significativos en los tres planos del espacio, especialmente si se combina con expansión rápida del maxilar. La mayoría de las maloclusiones clase III presentan deficiencia maxilar anteroposterior y vertical con una mandíbula que sobresale ligeramente y en promedio mordida cruzada anterior. Estos pacientes se manejan bien con expansión maxilar y máscara facial. Diversos estudios han demostrado que las fuerzas de protracción inducen a la separación de las suturas maxilares, remodelación ósea en suturas abiertas y varias respuestas en el complejo maxilofacial en sentido de la dirección de la protracción, ya que la estimulación de la actividad celular potencializa los resultados de la protracción ${ }^{11,12}$. Varias suturas circunmaxilares tiene un papel importante en el desarrollo del complejo nasomaxilar y se ven afectadas con éste tipo de tratamiento, ellas son: la sutura frontomaxilar, nasomaxilar, cigomáticotemporal, cigomáticomaxilar, pterigopalatina, intermaxilar, etmoidomaxilar y lacrimomaxilar.

Muchos autores reportan en sus estudios que el manejo de las maloclusiones clase III en edades tempranas no tiene estadísticamente cambios significativos como es el caso de Baik que dividió 47 niños coreanos en tres grupos: menores de 10 años, 10 a 12 años, y 12 años de edad o más.
No hubo diferencia estadísticamente significativa entre los tres grupos mediante la prueba de Kruskal-Wallis. El autor afirma, que "el número de cada grupo no fue suficiente para evaluar los efectos precisos de acuerdo a la edad." ${ }^{13}$ Merwin y col. examinaron 30 pacientes divididos en dos grupos: de 5 a 8 años de edad y 9 a 12 años de edad tratados con la expansión maxilar y máscara de Protracción y observaron que los cambios cefalométricos fueron similares entre los dos grupos de edad, lo que sugiere que la respuesta esqueletal fue similar en los pacientes, ya sea en dentición primaria o la dentición mixta tardía ${ }^{14}$.

Sin embargo, otros estudios han reportado una tendencia a un mayor cambio ortopédico en niños más pequeños. Baccetti y col. examinaron las diferencias entre el tratamiento temprano y el tratamiento tardío en dos grupos de niños tratados con expansión maxilar y máscara facial. El grupo más joven mostró avance significativamente mayor de las estructuras superiores, y el cóndilo tuvo un crecimiento más hacia arriba y hacia adelante como resultado del tratamiento ${ }^{15}$. Saadia y Torres, examinaron a 112 pacientes divididos igualmente en tres grupos de edad. Los resultados se obtuvieron más rápido y con menos horas de uso del aparato por día en los niños más pequeños ${ }^{16}$.

El Caso Clínico que presentamos tiene como propósito mostrar que el manejo temprano de las maloclusiones clase III tienen un mejor resultado y como el uso de la máscara de Protracción seguido de terapia ortopédica maxilar es de gran ayuda en estos pacientes.

La máscara tiene un arco anterior ajustable que se usa para hacer tracción del maxilar por medio de elásticos. En el maxilar superior se instala un aparato fijo de expansión rápida tipo Hirax soldado a bandas, o en medio de una férula de acrí- 
lico cementado a los dientes posteriores, en la región de los caninos este aparato presenta unos ganchos que servirán para conectar el maxilar al vástago de la máscara ${ }^{17}$.

El aparato de expansión se activa una vez al día durante un tiempo de entre 10 y 30 días dependiendo de la severidad del caso, cada activación de $0.25 \mathrm{~mm}$. Al tiempo que se realiza la expansión, se utilizan unos elásticos que proveen una fuerza en 300 y 600 gramos por cado lado y se recomienda el uso de la máscara por un promedio de 12 horas por día ${ }^{18,19}$.

Los estudios demuestran que la máscara facial y terapia de expansión palatina es un método eficaz para el tratamiento de las maloclusiones clase III y la intervención temprana da una mejor respuesta ortopédica ${ }^{20,21,22}$.

\section{Caso Clínico}

Paciente de 7 años de edad que acude al servicio de Odontología Pediátrica y Ortopedia Maxilar de la Escuela de Odontología de la Universidad del Valle (Cali-Colombia) acompañado por su madre la cual refiere como motivo de consulta "el niño tiene la mordida al revés". Se realizó la historia clínica general y odontológica y se diligencio el consentimiento informado no se hallaron antecedentes médicos de relevancia. Se

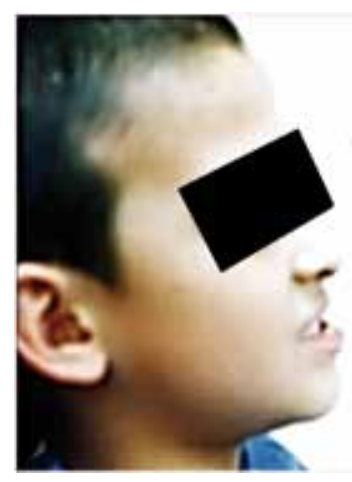

Fig. 1 a y b. Vista inicial de perfil e intraoral. encontró un niño de buen peso y buena talla, de ancestros indígenas, la madre del niño no es clara con los antecedentes familiares de prognatismo mandibular. En el examen clínico extraoral se observa un tipo braquifacial, dimensión vertical disminuida, leve asimetría facial (no significativa), perfil cóncavo, labios evertidos e incompetencia labial.

Intraoralmente presenta dentición mixta temprana sin caries, ni enfermedad periodontal con erupción de los cuatro primeros molares permanentes y de los incisivos inferiores, el 21 en proceso de erupción, relación molar y canina de clase III mordida cruzada anterior: overjet de $-4 \mathrm{~mm}$ y lengua baja y protrusiva (Fig.1 a y 1 b).

\section{Ayudas diagnósticas}

\section{$\underline{\text { Radiografía panorámica }}$}

En la radiografía panorámica inicial se observa senos paranasales maxilares sin ninguna alteración, tabique nasal desviado hacia la izquierda, cóndilos, cavidad glenoidea, ramas y cuerpo de la mandíbula en aparente normalidad, formación de todos los gérmenes permanentes a excepción de los terceros molares, alteración de la secuencia de erupción, el 11 en mesoversión y un supernumerario mesodiente que le impide la erupción (Fig. 2a).

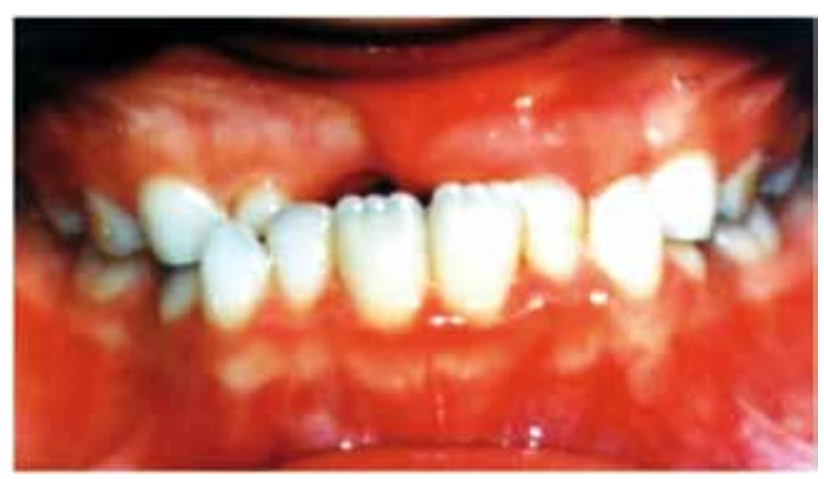




\section{$\underline{\text { Radiografía lateral de cráneo }}$}

La Tabla 1 muestra los trazos cefalométricos iniciales destacandose un Wits de $-3 \mathrm{~mm}$, una base craneal anterior corta, un ángulo ANB de $-3.5^{\circ}$, el tamaño total del maxilar (CO-A) y la altura facial inferior disminuidas, El punto A esta 3 mm por detrás de la perpendicular a Franfort desde Nasión datos que confirman una retrusión maxilar (Fig. 2b).

\section{Diagnóstico definitivo}

Paciente de sexo masculino de 7 años de edad, braquifacial, dimensión vertical disminuida, leve asimetría facial, perfil cóncavo, maloclusión clase III canina y molar, relación esqueletal
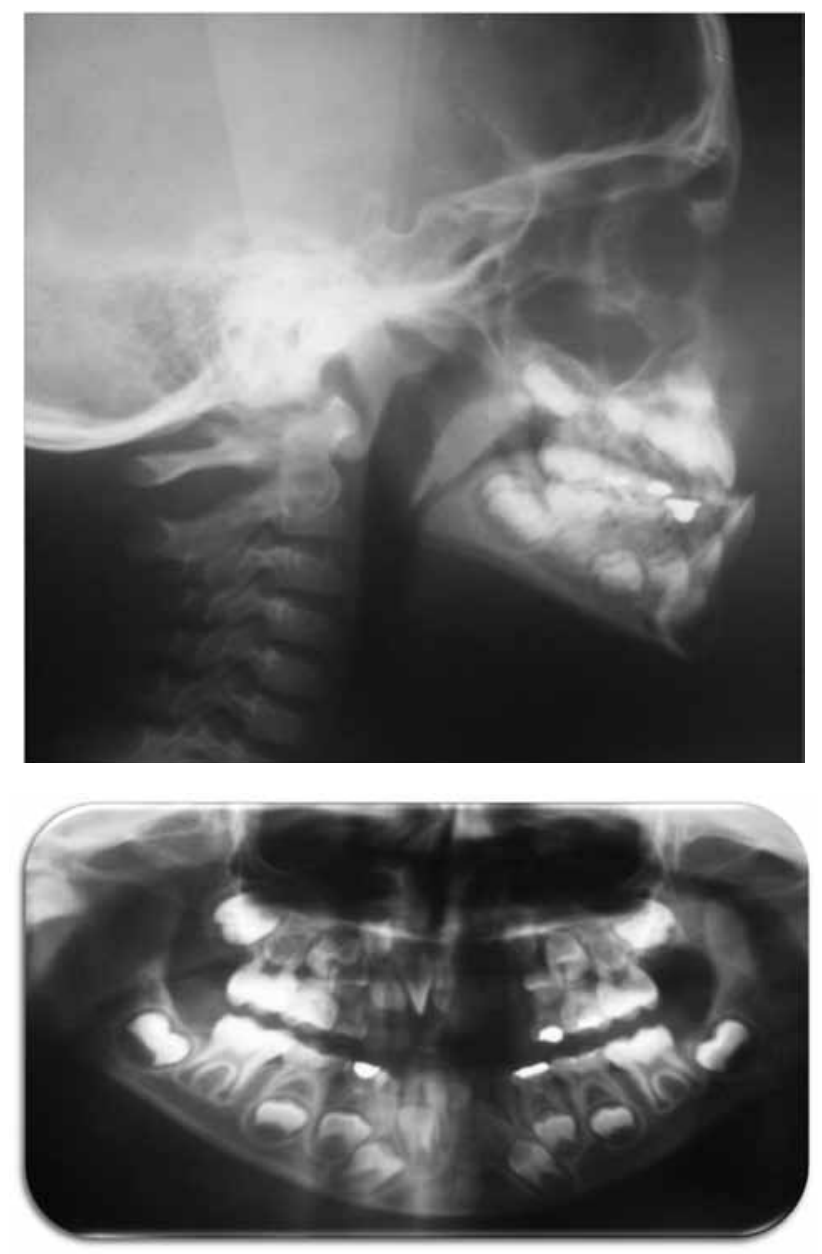

Fig. 2 a y b. Radiografías iniciales observese en la $R x p a-$ norámica la presencia de un mesodiente. clase III por retrusión maxilar, mordida cruzada anterior, overjet de $-4 \mathrm{~mm}$, lengua baja y protrusiva, mesodiente, malposición dentaria del 11 en giroversión.

\section{Plan de tratamiento}

\section{Fase I:}

Se remite para la exodoncia quirúrgica del supernumerario y posteriormente una vez los tejidos cicatrizados más o menos 3-4 meses se inicia el tratamiento con fonoaudiología para posicionamiento de la lengua y tratamiento ortopédico.

- Objetivos del tratamiento ortopédico: Interceptar maloclusión y relación esqueletal de clase III, armonizar perfil, corregir overjet y overbite además nivelar el plano oclusal.

\section{$\underline{\text { Fase II: }}$}

Reevaluación, guía de erupción y vigilancia de crecimiento para mantener los objetivos logrados.

\section{Secuencia de tratamiento}

La fase I se manejó con un tornillo tipo Hirax incluido en acrílico con ganchos en acero inoxidable por vestibular a nivel de los caninos, este dispositivo fue cementado a los dientes con ionómero de vidrio, el tornillo fue activado $1 / 4 \mathrm{de}$ vuelta 1 vez al día durante 7 días, tiempo necesario para la disyunción de las suturas. Pasados los 7 días se procede a realizar la tracción del maxilar con la máscara facial de protracción tipo petit con elásticos 4 1/2 oz 5/16" que se unían a los ganchos para protruir y una angulación de $30^{\circ}$ sobre el plano oclusal durante 6 meses, pasado este tiempo se aumenta la fuerza adicionando un elástico a cada lado, el dispositivo se usó 12 horas al día por un año (Fig. 3a y 3b). 
Tabla 1. Resultados cefalométricos obtenidos durante el tratamiento

\begin{tabular}{|c|c|c|c|c|}
\hline & NORMA & 7 AÑOS & 10 AÑOS & 13 AÑOS \\
\hline WITS & $0 \mathrm{~mm}$ & $-3 \mathrm{~mm}$ & $3 \mathrm{~mm}$ & $2 \mathrm{~mm}$ \\
\hline $\mathbf{S}-\mathbf{N}$ & $71 \mathrm{~mm}$ & $65 \mathrm{~mm}$ & $69 \mathrm{~mm}$ & $68 \mathrm{~mm}$ \\
\hline $\mathbf{S}-\mathbf{A r}$ & $32 \mathrm{~mm}$ & $30 \mathrm{~mm}$ & $32 \mathrm{~mm}$ & $36 \mathrm{~mm}$ \\
\hline$S-N-P F$ & $5.4^{\circ}$ & $3^{\circ}$ & $3^{\circ}$ & $2^{\circ}$ \\
\hline $\mathbf{S}-\mathbf{N}-\mathbf{A r}$ & $123^{\circ}$ & $118^{\circ}$ & $111^{\circ}$ & $116^{\circ}$ \\
\hline $\mathbf{S}-\mathbf{N}-\mathbf{A}$ & $80.6^{\circ}$ & $78.5^{\circ}$ & $87^{\circ}$ & $89^{\circ}$ \\
\hline $\mathbf{P F}-\mathbf{N}-\mathbf{A}$ & $88^{\circ}$ & $86^{\circ}$ & $89^{\circ}$ & $91^{\circ}$ \\
\hline ANB & $4.2^{\circ}$ & $-3.5^{\circ}$ & $3^{\circ}$ & $3^{\circ}$ \\
\hline Co-A & $85 \mathrm{~mm}$ & $78 \mathrm{~mm}$ & $84 \mathrm{~mm}$ & $85 \mathrm{~mm}$ \\
\hline $\mathbf{S}-\mathbf{N}-\mathbf{P P}$ & $8.5^{\circ}$ & $10^{\circ}$ & $7^{\circ}$ & $8^{\circ}$ \\
\hline$S-N-B$ & $76^{\circ}$ & $82^{\circ}$ & $81^{\circ}$ & $82^{\circ}$ \\
\hline $\mathbf{P F}-\mathbf{N}-\mathbf{P g}$ & $86.5^{\circ}$ & $87^{\circ}$ & $85^{\circ}$ & $88^{\circ}$ \\
\hline ENA-Me & $60-64 \mathrm{~mm}$ & $56 \mathrm{~mm}$ & $64 \mathrm{~mm}$ & $63 \mathrm{~mm}$ \\
\hline $\mathbf{S}-\mathbf{A r}-\mathrm{Go}$ & $3: 4$ & $3: 4$ & $3: 4$ & $3: 4$ \\
\hline $\mathbf{S}-\mathbf{N}-\mathbf{P M}$ & $34.2^{\circ}$ & $35^{\circ}$ & $35^{\circ}$ & $35^{\circ}$ \\
\hline Go-Me & $71 \mathrm{~mm}$ & $60 \mathrm{~mm}$ & $67 \mathrm{~mm}$ & $68 \mathrm{~mm}$ \\
\hline PF - PM & $26^{\circ}$ & $31^{\circ}$ & $34^{\circ}$ & $33^{\circ}$ \\
\hline Co-Pg & $97-100 \mathrm{~mm}$ & $98 \mathrm{~mm}$ & $108 \mathrm{~mm}$ & $112 \mathrm{~mm}$ \\
\hline$N(p)-A$ & $0 \mathrm{~mm}$ & $-3 \mathrm{~mm}$ & $-1 \mathrm{~mm}$ & $0 \mathrm{~mm}$ \\
\hline OVERJET & $2.5 \mathrm{~mm}$ & $-4 \mathrm{~mm}$ & $4 \mathrm{~mm}$ & $2 \mathrm{~mm}$ \\
\hline
\end{tabular}




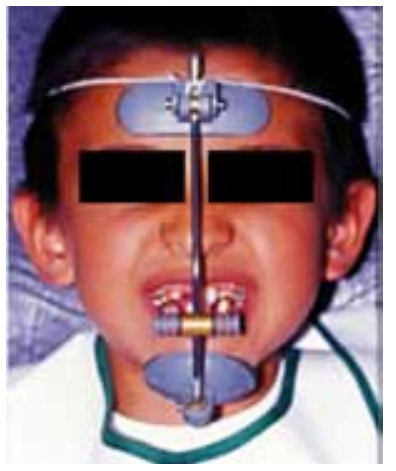

Fig. 3a. Paciente con Máscara de Protracción.

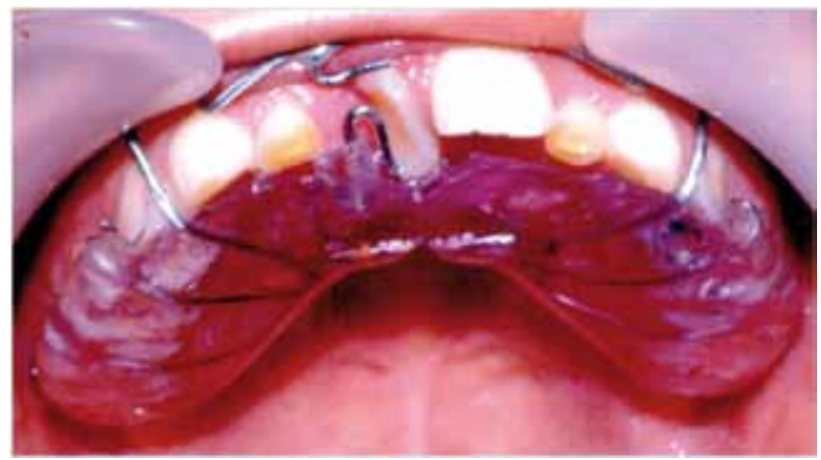

Fig. 4 a y b. Aparatología ortopédica funcional.

Al concluir la tracción se continuó el tratamiento con aparatología removible utilizando inicialmente Placa de Hawley y Arco de Eschler para retención Mandibular, una vez erupcionado el 11 se utiliza una Placa de Hawley con tornillo de expansión, planos posteriores y resorte en "S" para posicionamiento del 11 por un periodo de un año, se dedice cambiar aparatología para permitir el movimiento libre de los maxilares con unas pistas planas indirectas en mesoclusión y posteriormente un activador elástico con coffin sencillo y barra ondulada inferior que impide el contacto de la lengua con los dientes inferiores y arco de Eschler para contención mandibular por un periodo de un año y actualmente se encuentra en fase de contención (Fig. 4a, 4b y 5).

\section{Resultados}

Se tomaron 3 radiografías laterales de cráneo la primera antes del inicio del tratamiento con fi-

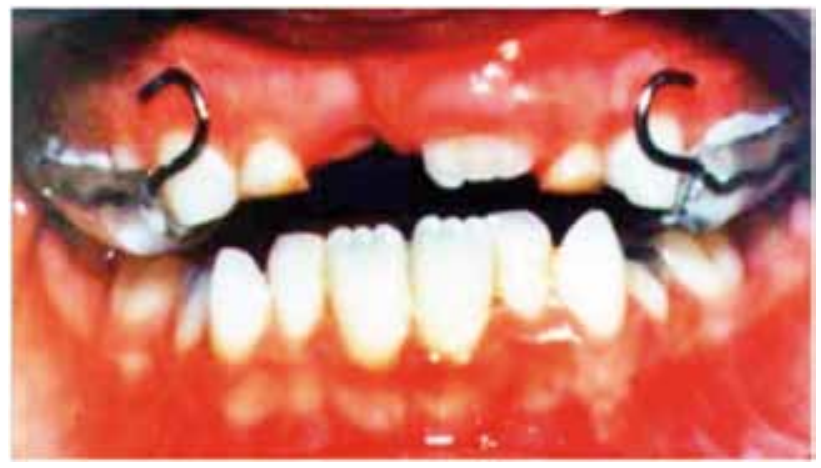

Fig $3 b$. Férula en acrilico para expansión transversal.
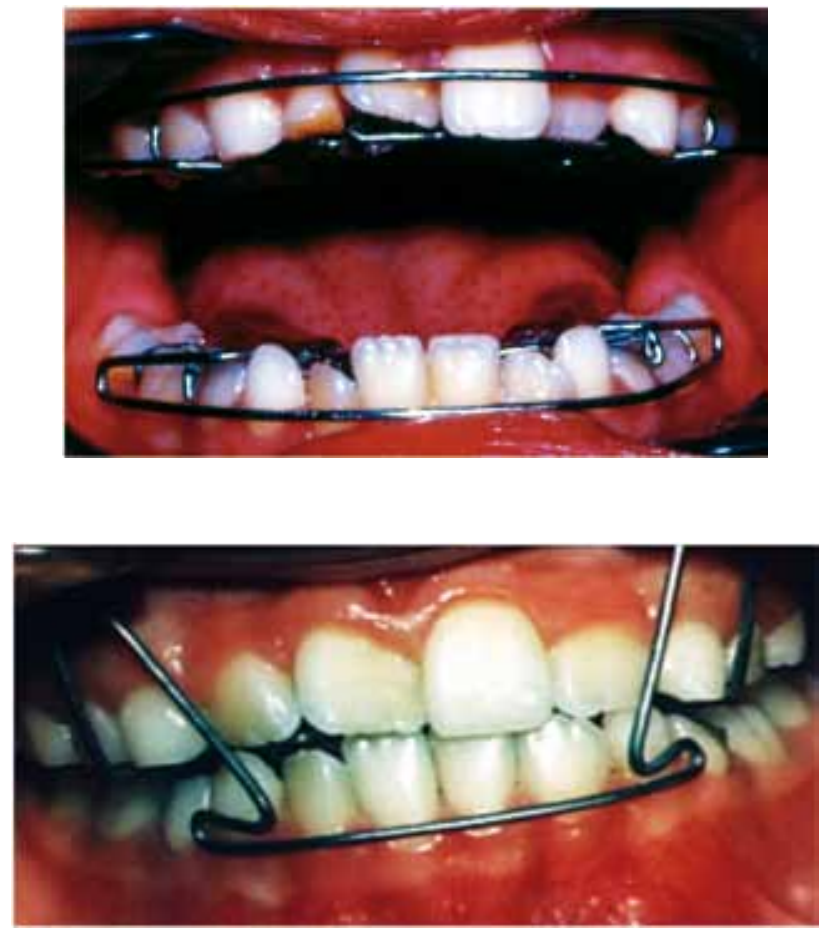

Fig. 5. Activador Abierto modificado con arco de Progenie.

nes diagnósticos tomadas en intervalos de cada 3 años. Se observó a los 7 años un Wits de -3 $\mathrm{mm}$, a los 10 años $3 \mathrm{~mm}$ y a los 13 años $2 \mathrm{~mm}$, hubo un aumento en la profundidad de la cara que se evidencia por el incremento de las medidas: base craneal anterior, longitud de la cara media (Co-A), longitud del cuerpo mandibular (Go-Me), también se presentaron cambios en el maxilar por aumento del ángulo de Landhe, el punto A a la perpendicular Nasión y en la longitud de la cara media lo que demuestra que presentó un adelantamiento maxilar y a nivel 
mandibular se observó que hubo un crecimiento estable y controlado (Fig. 6, 7 y 8) y Tabla 1. por lo tanto se comprueba que el tratamiento temprano con la máscara facial de Protracción y expansión maxilar ayuda a prevenir cambios progresivos irreversibles en tejidos duros $y$ blandos (Fig. 9) como lo confirma Nihat Kilic y col. en un estudio de casos y controles donde compara 24 niñas con maloclusión clase III y 15 niñas de grupo control clase I donde al inicio del estudio se observa una retrusión de los tejidos blandos del perfil facial, al terminar el tratamiento se observó que el maxilar y los tejidos blandos circundantes muestran un significativo
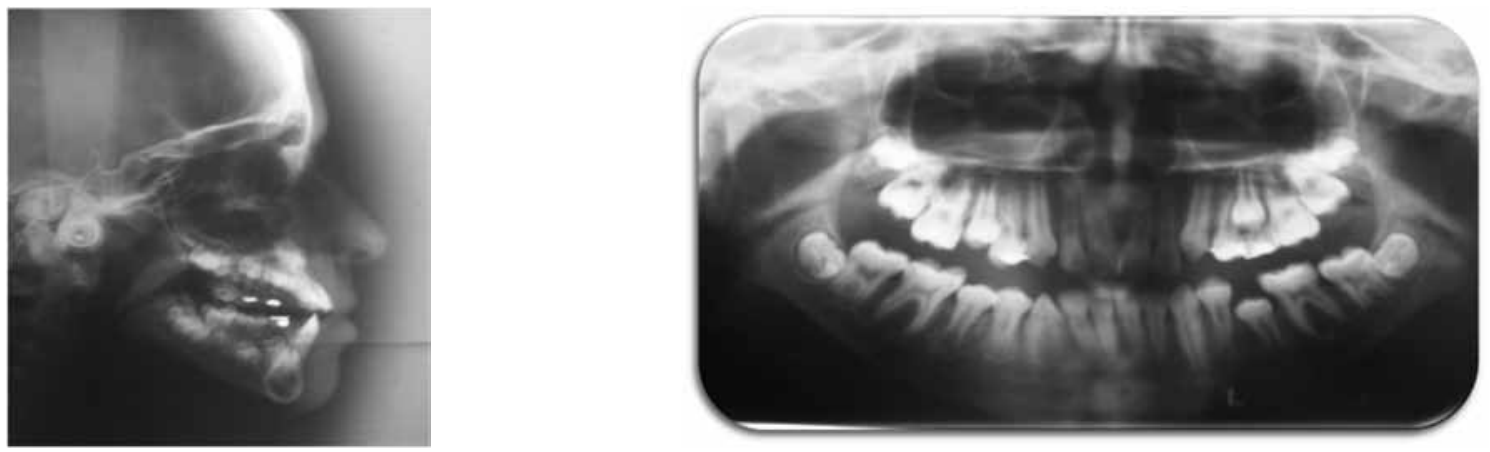

Fig. 6 a y b. Radiografías Lateral de Cráneo y Panorámica tomadas 3 años después de iniciado el tratamiento.
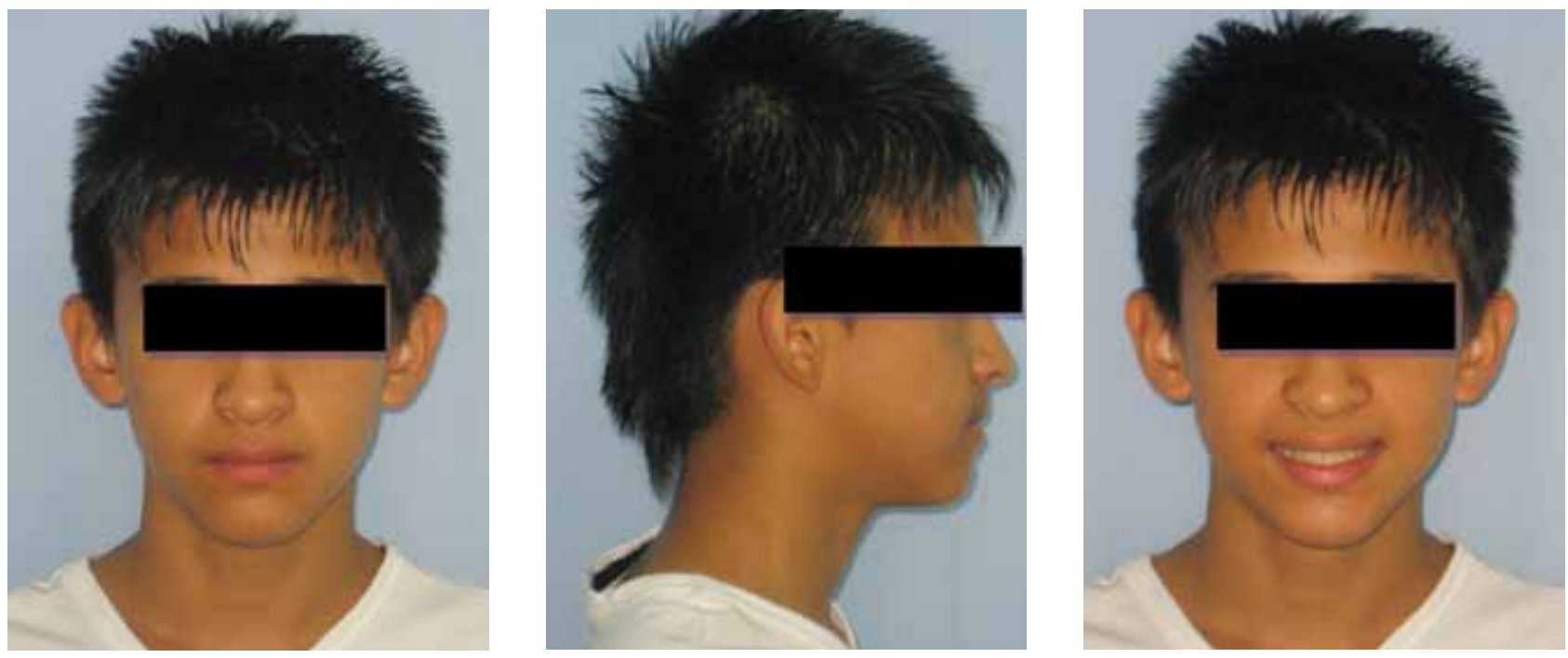

Fig. 7 a - c. Fotografías frontales y de perfil al término del tratamiento.
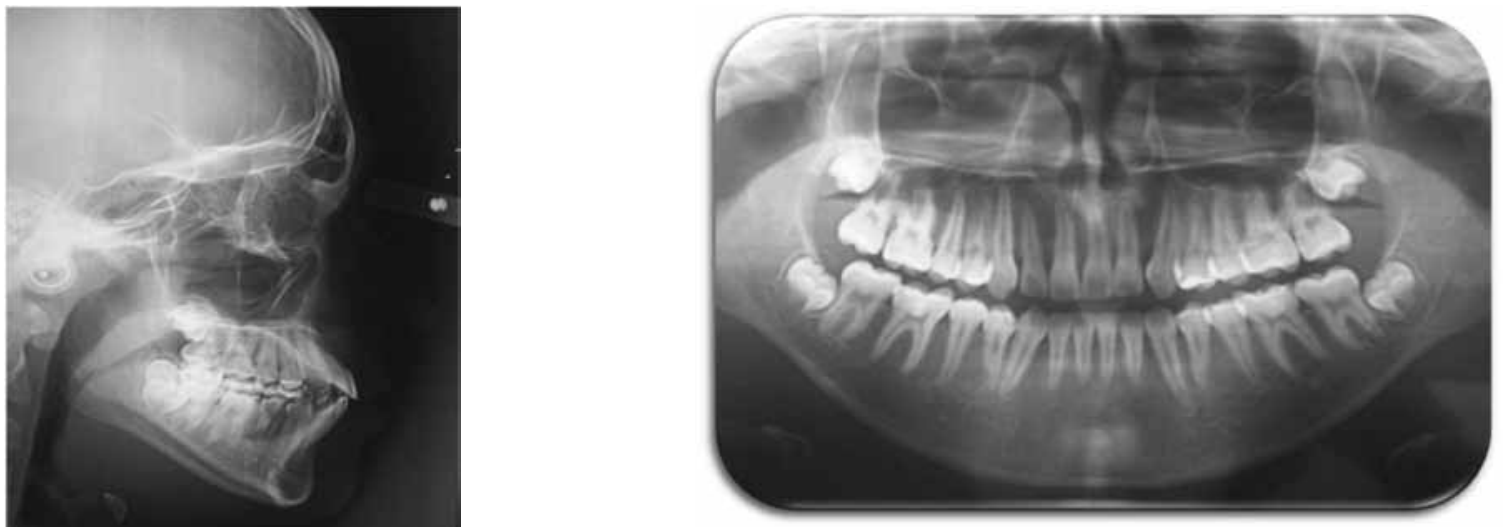

Fig. 8 a y b. Radiografías Lateral de Cráneo y Panorámica tomadas a los 13 años de edad. 


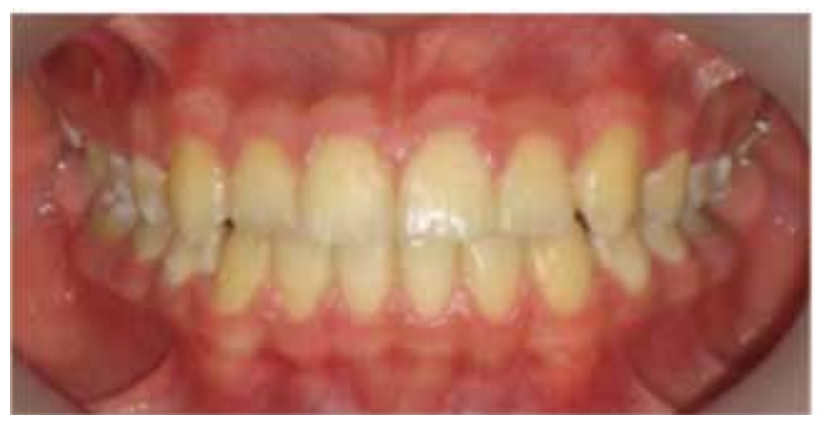

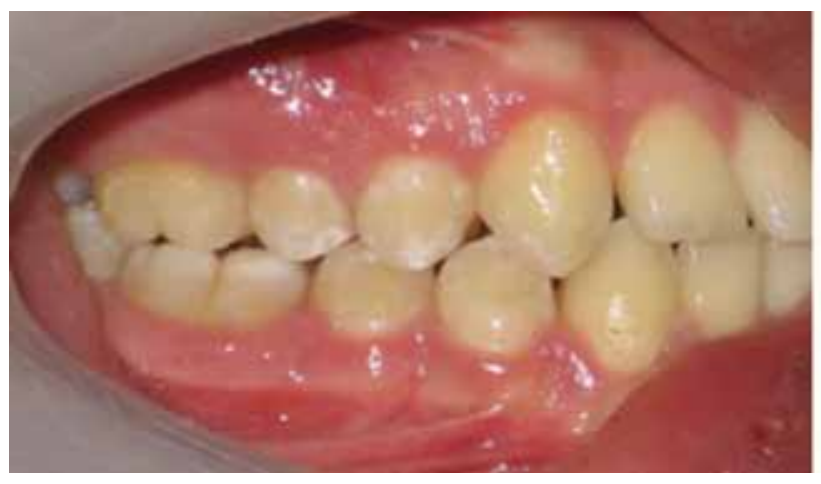

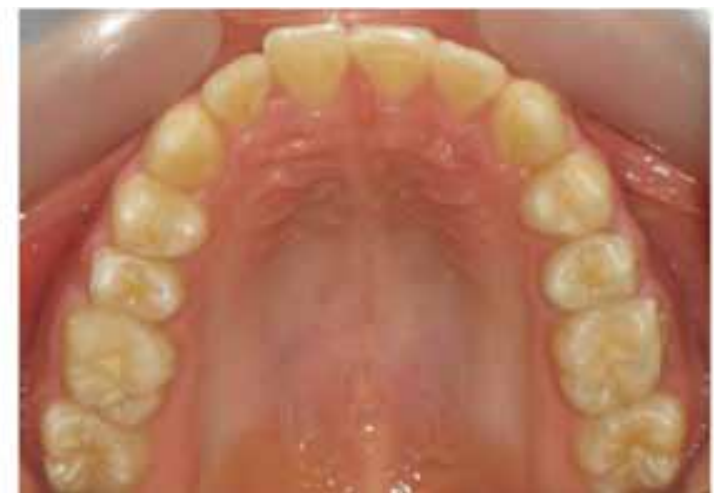

Fig. $9 a-e$. Fotografías intraorales al final del tratamiento.

movimiento anterior y como la mandíbula y los tejidos blandos mostraron un retroceso y rotación hacia abajo. Esta mejora del perfil se dio por los cambios de los tejidos blandos del maxilar y los cambios en los tejidos duros de la mandíbula2 $2^{0,23,24}$. Contrario a lo que dice Ayca Arman, T ufuk Toyga donde afirma que las características de los pacientes con maloclusión clase III siguen presentes así estos pacientes sean tratados ${ }^{25}$. En el caso clínico reportado podemos observar el mejoramiento del perfil y la armonización de los tejidos duros y blandos; se comprueba como el uso de la máscara también mejora las discre-
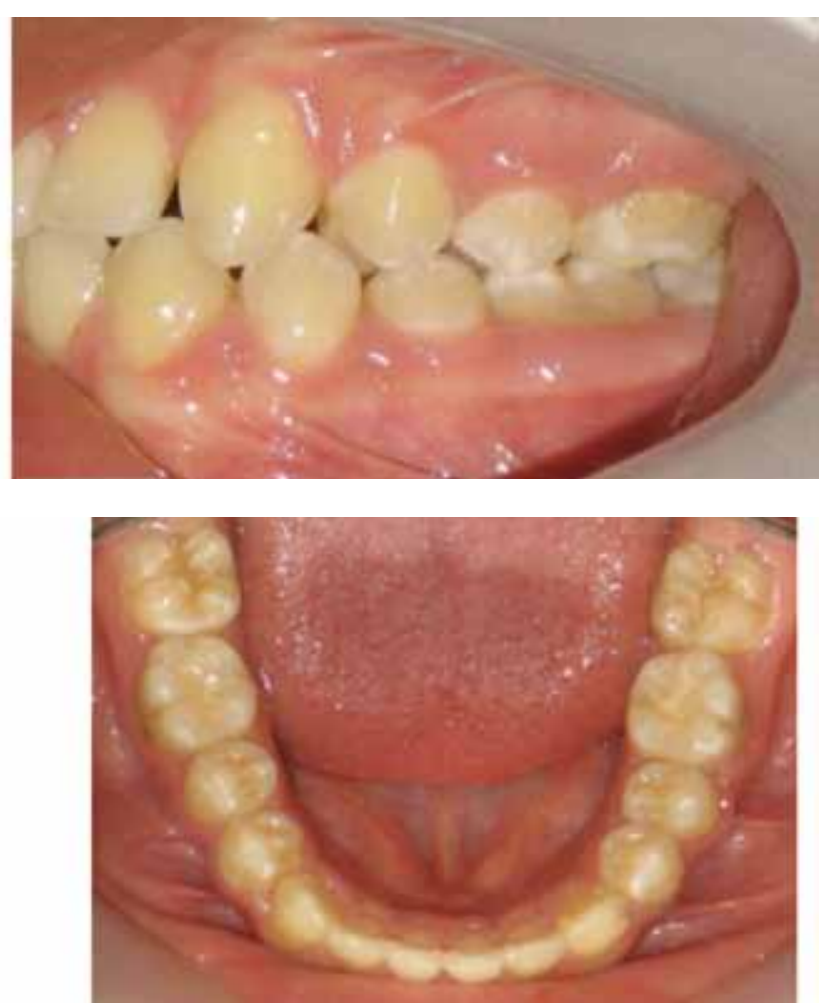

pancias esqueléticas y provee mejores condiciones para el futuro crecimiento, optimizando la función oclusal y disminuyendo la posibilidad de cirugía ortonágtica, favoreciendo la estética facial del niño ${ }^{26}$.

\section{Conclusiones}

- Este caso clínico muestra la evolución favorable del tratamiento temprano de la maloclusión clase III utilizando terapia combinada de ortopedia extraoral y aparatología ortopédica maxilar. 
- Gran parte del éxito se basa en un preciso diagnóstico y una colaboración adecuada por parte del paciente y su familia.

- La terapéutica temprana trae cambios favorables en tejidos duros y blandos, mejora las relaciones espaciales de los maxilares y las funciones del sistema estomatognatico, trae igualmente beneficios sicológicos en estos pacientes.

\section{Referencias}

1. Yagci A, Uysal T . Efecto de la terapia con mascara facial convencional o modificada en la posición condilar en pacientes con Maloclusión clase III. Orthod craniofac Res (2010); 13: 246-254.

2. Tsai H-H: Components of anterior crossbite in the primary dentition. Journal Dent Child (2001); 27-32.

3. Fengshan C, Kazuto T, Luyi Y and Isao S. Arch widths and mandibular- maxillary base widths in class III malocclusions from ages 10 to 14. American Journal of Orthodontics and Dentofacial Orthopedics. (2008) ; 65-69.

4. Miyajima K, McNamara JA Jr., Sana M, Murata S. An estimation of craniofacial growth in the untreated Class III female with anterior crossbite. American Journal of Orthodontics and Dentofacial Orthopedics. (1997): 112:425-34.

5. Thilander B, Pena L, Infante C, Parada SS, de Mayorga C. Prevalence of malocclusion and orthodontic treatment need in children and adolescents in Bogota, Colombia. An epidemiological study related to different stages of dental development. European Journal of Orthodontics (2001); 23:153-67.

6. Patrick K. Treatment of the Class III Malocclusion with maxillary expansión and protraction. Seminars in Orthodontics. (2007) 13; 3: 143-157.

7. Hernández J, Soto L. La Máscara facial de protracción en el tratamiento temprano de la maloclusión Clase III. Revista Estomatología, (2006) 14: 6-11 .

8. Simoes W. Ortopedia Funcional de los Maxilares. 3ed. Sao Paulo: Artes Médicas; (2004): 208-215.

9. Gu Y, Rabie AB, Hagg U. Treatment effects of simple fixed appliance and reverse headgear in correction of anterior crossbites. American Journal of Orthodontics and Dentofacial Orthopedics. (2000);117:691-9.

10. Jane Mermigos CAF, George Andreasen. Protraction of the maxillofacial complex. American Journal of Orthodontics and Dentofacial Orthopedics. (1990);98:47-55.

11. Oltramari PVP. Tratamento ortopédico da Classe III em padroes faciais distintos. R Dental Press Ortodon Ortop Facial. (2005);10:72-82.

12. Da Silva de Carballo L. Tratamiento de la maloclusión de clase III con máscara facial. Acta Odontológica Venezolana. (2006); 44:424-430. Disponible en la World WideWeb:<http:/ / www.scielo.org.ve/ scielo.php?script=sci_arttext\&pid=S0001$63652006000300022 \& \operatorname{lng}=\mathrm{es} \& n r m=\mathrm{iso}>$. ISSN 0001-6365.

13. Baik HS. Clinical results of maxillary protraction in Korean children. American Journal of Orthodontics and Dentofacial Orthopedics. (1995); 108: 583-592.

14. Merwin D, Ngan P. Hagg U. Timing for effective application of anteriorly directed orthopedic force to maxilla. American Journal of Orthodontics and Dentofacial Orthopedics. (1997); 112: 292-299.

15. Baccetti T, McGill JS, Franchi L. Skeletal effects of early treatment of Class III malocclusion with maxillary expansion and face-mask therapy. American Journal of Orthodontics and Dentofacial Orthopedics. (1998); 113: 333-343.

16. Saadia M, Torres E. Sagittal changes after maxillary protraction with expansion in Class III patients in the primary, mixed, and late mixed dentitions. American Journal of Orthodontics and Dentofacial Orthopedics. (2000); 117: 669-680.

17. Gallagher W, Miranda F, Buschang PH: Maxillary protraction: treatment and post-treatment effects. American Journal of Orthodontics and Dentofacial Orthopedics. (1995); 113: 612-619.

18. Franchi L, Baccetti T, Mcnamara JA Jr: Shape-coordinate analysis of skeletal changes induced by rapid maxillary expansion and facial mask therapy. American Journal of Orthodontics and Dentofacial Orthopedics. (1998); 114: 418- 426. 
19. Yoshida I, Takahiro S and Itaru M. Effects of treatment with a combined maxillary protraction and chincap appliance in skeletal Class III patients with different vertical skeletal morphologies. European Journal of Orthodontics. (2007); 29: 126-133.

20. Nihat K, Gulhan C, Ali K and Hüsamettin O. Soft tissue profile changes following maxillary protraction in Class III subjects. European Journal of Orthodontics. (2010); 32: 419-424.

21. Espinal G. Betancourt J. Evaluación de los cambios esqueléticos y dentales producidos con el tratamiento con placas de acetato y elásticos de Clase III en pacientes con mesio-relación esquelética y dental en dentición primaria y mixta temprana. Revista Facultad de Odontología Universidad de Antioquia, (2005); 16: 71-80.

22. Yasar G, Seyda E. The effect of modified reverse headgear force applied a facebow on the dentofacial structures. European Journal of Orthodontics. (2004); 26: 51-57.

23. Baccetti T, Rey D, Angel D, Oberti G, James A. McNamara Jr.Mandibular Cervical Headgear vs Rapid Maxillary Expander and Facemask for Orthopedic Treatment of Class III Malocclusion. Angle Orthodontist. (2007); 77: 619- 624.

24. Yawaka Y, Hironaka S, Akiyama A, Matzuduka I, Takasaki C, Oguchi H. Changes in occlusal contact area and average bite pressure during treatment of anterior crossbite in primary dentition. Journal Clin Pediatr Dent. (2003); 28:75-9.

25. Ayca Arman, T Ufuk Toyga y Eyas Abuhijleh. Evaluation of maxillary protraction and fixed appliance therapy in Class III patiens. European Journal of Orthodontics (2006); 28: 383-392.

26. Rospigliosi W, Torres G, Soldevilla L, Coronado M. Reporte de caso: Tratamiento de Maloclusion Clase III en Denticion Mixta temprana con Expansion Rapida de Maxila y Mascara Facial. Universidad mayor de San Marcos . Unidad de Posgrados de la Facultad de Odontología. (2011);2: 1-14.

Recibido 19-10-2011

Aceptado: 06-11-2011

Correspondencia: angelicampabon@hotmail.com 